\title{
Gastrointestinal Manifestations in Hereditary Transthyretin Amyloidosis: a Single-Centre Experience
}

\author{
Marco Luigetti ${ }^{1,2}$, Annalisa Tortora ${ }^{2,3}$, Angela Romano ${ }^{2,4}$, Andrea Di Paolantonio ${ }^{2}$, Valeria Guglielmino ${ }^{1,2}$, Giulia $^{2}$ \\ Bisogni $^{4}$, Antonio Gasbarrini ${ }^{2,3}$, Paolo Calabresi ${ }^{1,2}$, Mario Sabatelli ${ }^{2,4}$
}

1) Fondazione Policlinico

Universitario A. Gemelli IRCCS, UOC Neurologia, Roma;

2) Università Cattolica del Sacro Cuore, Roma;

3) Fondazione Policlinico

Universitario A.

Gemelli IRCCS, UOC

Gastroenterologia, Roma;

4) Centro Clinico NEMO

adulti, Roma, Italia

\author{
Address for correspondence: \\ Marco Luigetti, MD \\ UOC Neurologia \\ Largo A Gemelli 8, 00168 \\ Rome, Italy \\ mluigetti@gmail.com
}

\begin{abstract}
Background \& Aims: Hereditary transthyretin (ATTRv) amyloidosis represents a diagnostic challenge considering the great variability in clinical presentation and multiorgan involvement. In this study we report the prevalence of gastrointestinal (GI) involvement of patients with hereditary ATTRv amyloidosis from one single center of Italy, a non-endemic area.

Methods: We retrospectively analyzed a cohort of 39 patients with hereditary ATTRv amyloidosis followed at the Neurology Unit of Fondazione Policlinico Universitario A. Gemelli IRCCS in Rome, Italy. All patients had a documented mutation in the gene encoding the thansthyretin. Neurological, cardiological and gastrointestinal manifestations were systematically collected at every monitoring visit.

Results: $82 \%$ reported at least one GI symptom. Unintentional weight loss was the most frequently reported. Lower GI symptoms were more frequent than upper GI symptoms (66.7\% vs. $35.9 \%, \mathrm{p}=0.0122)$. The first GI symptom was always reported within 5 years since disease onset. Gastrointestinal symptoms were almost always present in patients with Val30Met mutation $(93.8 \%, 15 / 16)$, and in more than half of the cases with Phe64Leu mutation $(66.7 \%, 8 / 12)$. All cases with a non-Val30Met mutation disclosed almost all GI symptoms within 5 years since disease onset; conversely, patients with Val30Met mutation continued to develop further GI manifestations during the disease course.

Conclusions: Prevalence of GI symptoms in our cohort was $82 \%$, resulting in a higher prevalence than reported in the THAOS registry. Gastroenterologists, therefore, play an important role for the management of the disease, and their expertise should be valued for an effective multidisciplinary approach to this condition.
\end{abstract}

Key words: amyloid transthyretin - hereditary ATTRv amyloidosis - diarrhea - neuropathy - sudoscan.

Abbreviations: ATTRv: amyloid transthyretin, v for variant; FAP: familial amyloidotic polyneuropathy; GI: gastrointestinal; TTR: transthyretin.

\section{INTRODUCTION}

Hereditary transthyretin (ATTR) amyloidosis is a rare disease due to mutations in the gene encoding transthyretin (TTR) and characterized by multisystem extracellular deposition of amyloid, leading to a dysfunction of different organs and tissues [1]. To date, more than 100 TTR variants have been described as a cause of hereditary ATTRv amyloidosis, the most frequent being the Val30Met mutation [2].
Hereditary ATTRv amyloidosis represents a diagnostic challenge to clinicians considering the great variability in the clinical presentation and multiorgan involvement. Generally, patients present with polyneuropathy, but clinicians should consider the frequent cardiac, ocular, and gastrointestinal (GI) impairment $[1,3,4]$. However, the pattern of the clinical impairment may vary according to the geographic areas. In the endemic areas, patients present with an early-onset (third to fourth decade) disease and deteriorate quickly because of autonomic dysfunction and rapid progression of the sensory-motor deficit [5]. Conversely, in non-endemic areas, many patients present with a late-onset disease (age of $\geq 50$ years at symptom onset), and the polyneuropathy (affecting predominantly the large nerve fibres) progresses slowly, often with cardiac involvement but with less autonomic dysfunction $[6]$. 
Gastrointestinal manifestations are commonly reported in patients with hereditary ATTRv amyloidosis $[7,8]$ representing a frequent cause for a poor quality of life [9]. Gastrointestinal symptoms can anticipate the onset of the polyneuropathy, and the most commonly reported initial symptoms are often diarrhea, unintentional weight loss, constipation or nausea [7].

Current therapeutic options increase the survival of patients, slowing the progression of the polyneuropathy and/ or cardiomyopathy [10-13]. No therapy is approved for the management of GI disturbances, even if some effects of the available drugs have been described [14].

In the present study, we report the prevalence of GI involvement in a cohort of patients with hereditary ATTRv amyloidosis, with different mutations from Italy, a nonendemic area.

\section{METHODS}

This study was approved by the Ethics Committee of the Università Cattolica del Sacro Cuore di Roma in accordance with the Declaration of Helsinki. All patients included in the study signed an informed consent.

We recruited 39 patients with hereditary ATTRv amyloidosis associated with different mutations, regularly followed up in our University hospital. The identification of a documented TTR mutation through DNA analysis was required for the diagnosis of hereditary ATTRv amyloidosis.

Symptoms were reported as present or absent, at the time of the last visit. Symptoms duration was calculated retrospectively from information provided by the patients. Cardiomyopathy was defined if a septum thickness $>13 \mathrm{~mm}$ was found on the echocardiography. To evaluate the extent of disability we performed every six month a comprehensive clinical neurological assessment (reflexes, sensation, and muscle weakness). The stage of neurological disability was defined according to familial amyloidotic polyneuropathy (FAP) scale [15].

The reported GI symptoms included the following items: early satiety, nausea, vomiting, diarrhea, constipation, alternating diarrhea/constipation, fecal incontinence, abdominal pain, abdominal bloating, and unintentional weight loss.

We did not routinely perform an instrumental or laboratory diagnostic work-up in our cohort. Some patients occasionally underwent gastroscopy, colonoscopy, abdominal ultrasound, or an abdominal CT scan in order to detect any cause of symptoms different from ATTRv. Data regarding symptomatic treatment were also collected.

Statistical analysis of data was performed by SPSS (Statistical Package for Social Science) version 24.0. The chisquare test was used for categorical variables. Significance $p$ value was set at 0.05 .

\section{RESULTS}

A total of 39 patients (mean age at onset 63.1 years; 29 males) with hereditary ATTRv amyloidosis were enrolled in the study. Neuropathy was observed in $92.3 \%(36 / 39)$ of patients; $50 \%(18 / 36)$ of them were in the stage I of the disease according to the FAP scale, $33.3 \%(12 / 36)$ in stage II, and $16.6 \%(6 / 36)$ in stage III. Cardiomiopathy was detected in $56.4 \%(22 / 39)$ of patients. The mean disease duration was $82.4 \pm 48.4$ months. Thirty-three patients were in treatment for the disease: 19 with Tafamidis, 6 with Patisiran, 5 with Diflunisal and 3 with Inotersen.

Characteristics of patients with hereditary ATTRv amyloidosis associated with different mutations and the distribution of GI symptoms are summarized in Table I.

At least one GI symptom was reported by $82.1 \%(32 / 39)$ of patients. Unintentional weight loss was the most frequently reported symptom $(46.2 \%, 18 / 39)$. Other common symptoms were: diarrhea $(35.9 \%, 14 / 39)$, abdominal bloating $(28.2 \%$, $11 / 39)$, alternating diarrhea/constipation (25.6\%, 10/39), early satiety $(23.1 \%, 9 / 39)$ and constipation $(20.5 \%, 8 / 39)$. Conversely, the following symptoms occurred rarely: nausea $(5 / 39,12.8 \%)$, vomiting $(4 / 39,10.3 \%)$, fecal incontinence $(3 / 39$, $7.7 \%)$, and abdominal pain $(3 / 39,7.7 \%)$.

Overall, lower GI symptoms were more frequent than upper GI symptoms ( $66.7 \%$ vs. $35.9 \%, p=0.0122)$; these results were not confirmed dividing patients based on TTR variant, being not statistically significant neither for Val30Met (75.0\% vs. $37.5 \% \mathrm{p}=0.0732$ ) nor for Phe64Leu (41.7\%, vs. $33.3 \%, \mathrm{p}=1)$ cases.

No significant gender related difference in overall GI symptom prevalence was found (females $80.0 \%$, vs. males $82.8 \%)$. Only unintentional weight loss was significantly more common in females than in males $(80.0 \%$, vs. $34.5 \%$; $\mathrm{p}=0.025)$.

Gastrointestinal symptoms were almost always present in patients with Val30Met mutation $(93.8 \%, 15 / 16)$, and in more than half of cases with Phe64Leu mutation (66.7\%, 8/12). Unintentional weight loss was the most common symptom also grouping patients by a single mutation. Interestingly, abdominal bloating was reported by a great proportion of patients with Val30Met $(43.8 \%, 7 / 16)$.

Considering the onset of GI symptoms, the first symptom was always reported within 5 years from disease onset (Table II). Grouping patients by TTR variant, patients with Val30Met mutation continued to develop further GI manifestations over time, in one case even 10 years after the disease onset, with an increased prevalence of different GI symptoms during the disease course. Conversely, all non-Val30Met cases disclosed almost all GI symptoms within 5 years since disease onset, without any increase of GI symptoms prevalence during the disease course (Table II).

Considering the entire cohort, the mean time of onset of the first GI symptom since disease onset was 36.71 months (standard deviation: 30.51). Conversely, regarding the same parameter, we observed the following data dividing patients based on TTR variant: Val30Met 49.56 months (standard deviation: 37.91); Phe64Leu 25.71 months (standard deviation: 14.57); Glu89Gln 20.0 months (standard deviation:6.93); Ala120Ser 15.96 months (standard deviation:6.93); Tyr59Lys 11 months; Ala109Ser 59 months.

Abdominal ultrasound was performed in 8 patients ( 4 Val30Met; 2 Phe64Leu; 1 Ile88Leu; 1 Val122Leu); abdominal CT scan was performed in 5 patients (1 Val30Met; 3 Phe64Leu; 1 Ala109Ser); colonoscopy was performed in 4 patients (1 Val30Met; 3 Phe64Leu); gastroscopy was performed in 1 
Table I. Characteristics of patients with hereditary transthyretin amyloidosis (ATTRv).

\begin{tabular}{|c|c|c|c|c|c|c|c|c|c|}
\hline & Val30Met & Phe64Leu & Glu89Gln & Ala120Ser & Thr59Lys & Ala109Ser & Val122Ile & Ile88Leu & Total \\
\hline Number of patients & 16 & 12 & 4 & 3 & 1 & 1 & 1 & 1 & 39 \\
\hline Male/Female & $14 / 2$ & $10 / 2$ & $1 / 3$ & $0 / 3$ & $1 / 0$ & $1 / 0$ & $1 / 0$ & $1 / 0$ & $29 / 10$ \\
\hline Early-onset (<50 years) & $1 / 16$ & - & - & - & $1 / 1$ & - & - & - & $2 / 39$ \\
\hline Late-onset ( $\geq 50$ years) & $15 / 16$ & $12 / 12$ & $4 / 4$ & $3 / 3$ & - & $1 / 1$ & $1 / 1$ & $1 / 1$ & $37 / 39$ \\
\hline Mean age at onset (years) & 64.3 & 64.5 & 53.3 & 64.0 & 47 & 65 & 79 & 62 & 63.1 \\
\hline Mean disease duration (months) & 90 & 71 & 89 & 80 & 132 & 144 & 12 & 36 & 82.4 \\
\hline Disease duration ( $<5$ years $)$ & $5 / 16$ & $6 / 12$ & $1 / 4$ & $1 / 3$ & - & - & $1 / 1$ & $1 / 1$ & $15 / 39$ \\
\hline Disease duration (5-10 years) & $4 / 16$ & $3 / 12$ & $1 / 4$ & $1 / 3$ & - & - & - & - & $9 / 39$ \\
\hline Disease duration ( $>10$ years) & $7 / 16$ & $3 / 12$ & $2 / 4$ & $1 / 3$ & $1 / 1$ & $1 / 1$ & - & - & $15 / 39$ \\
\hline Any gastro-intestinal symptoms & $15 / 16$ & $8 / 12$ & $3 / 4$ & $3 / 3$ & $1 / 1$ & $1 / 1$ & $0 / 1$ & $1 / 1$ & $32 / 39$ \\
\hline Unintentional weight loss & $6 / 16$ & $6 / 12$ & $3 / 4$ & $3 / 3$ & - & - & - & - & $18 / 39$ \\
\hline Nausea & $4 / 16$ & $1 / 12$ & - & - & - & - & - & - & $5 / 39$ \\
\hline Early satiety & $3 / 16$ & $3 / 12$ & - & $2 / 3$ & $1 / 1$ & - & - & - & $9 / 39$ \\
\hline Vomiting & $2 / 16$ & $1 / 12$ & - & - & $1 / 1$ & - & - & - & $4 / 39$ \\
\hline Diarrhea & $3 / 16$ & $4 / 12$ & $3 / 4$ & $2 / 3$ & $1 / 1$ & $1 / 1$ & - & - & $14 / 39$ \\
\hline Constipation & $3 / 16$ & $1 / 12$ & - & $2 / 3$ & $1 / 1$ & - & - & $1 / 1$ & $8 / 39$ \\
\hline Diarrhea/constipation & $4 / 16$ & $2 / 12$ & - & $3 / 3$ & $1 / 1$ & - & - & - & $10 / 39$ \\
\hline Fecal incontinences & $0 / 16$ & $2 / 12$ & - & - & - & $1 / 1$ & - & - & $3 / 39$ \\
\hline Abdominal pain & $2 / 16$ & $0 / 12$ & - & - & - & - & - & $1 / 1$ & $3 / 39$ \\
\hline Abdominal bloating & $7 / 16$ & $1 / 12$ & - & 1 & $1 / 1$ & - & - & $1 / 1$ & $11 / 39$ \\
\hline Peripheral Neuropathy & $13 / 16$ & $12 / 12$ & $4 / 4$ & $3 / 3$ & $1 / 1$ & $1 / 1$ & $1 / 1$ & $1 / 1$ & $36 / 39$ \\
\hline Stage I & $4 / 16$ & $9 / 12$ & $1 / 4$ & $1 / 3$ & $1 / 1$ & - & $1 / 1$ & $1 / 1$ & $18 / 36$ \\
\hline Stage II & $6 / 16$ & $2 / 12$ & $3 / 4$ & - & - & $1 / 1$ & - & - & $12 / 36$ \\
\hline Stage III & $3 / 16$ & $1 / 12$ & - & $2 / 3$ & - & - & - & - & $6 / 36$ \\
\hline Cardiac involvement & $10 / 16$ & $4 / 12$ & $2 / 4$ & $3 / 3$ & $1 / 1$ & $0 / 1$ & $1 / 1$ & $1 / 1$ & $22 / 39$ \\
\hline
\end{tabular}

patient (1 Phe64Leu). All tests failed to detect any cause of symptoms different from hereditary ATTRv amyloidosis.

Symptomatic treatment was used as following: domperidone in 3 patients (2 Val30Met; 1 Glu89Gln); loperamide in 3 patients (2 Val30Met; 1 Ala109Ser); lactulose in 1 patient (Phe64Leu); octreotide in 1 patient (Ala109Ser); nutritional supplementation in 5 patients (1 Val30Met; 3 Phe64Leu; 1 Ala120Ser).

\section{DISCUSSION}

Gastrointestinal symptoms are generally common in hereditary ATTRv amyloidosis and often occur early after disease onset [7]; our results confirm this data. Early recognition of GI dysfunction is important to enable prompt treatment to relieve symptoms, preserve quality of life, and prevent malnutrition [16]. Diagnostic tools for GI abnormalities are still limited, so currently self-reported symptoms are widely accepted measures for monitoring GI involvement in hereditary ATTRv amyloidosis $[7,8,16]$.

Prevalence of GI symptoms in our cohort was $82 \%$, resulting higher than prevalence reported in the largest analysis formulated based on data from THAOS registry (59\%) [7], and then prevalence observed in the general population (25-30\%) [16]. Probably, our study, being based on targeted questions, could overestimate the prevalence of GI symptoms while a registry study, such as THAOS, could underestimate their prevalence, considering that the registration procedure is not identical across sites, and many different specialists may be involved.

Globally, we did not find any gender prevalence for GI symptoms. Only unintentional weight loss was more frequent in females, contrary to what was reported in the THAOS registry [7].

In our cohort, GI manifestations appear early in the disease course: mean onset of the first GI symptom was 3 years from disease onset, and all patients with non-Val30Met mutations developed almost all GI symptoms within 5 years from the onset.

Regarding Val30Met, the onset of GI symptoms was equally distributed within 5 years, and between 5 and 10 years, from the onset, with an increase of global GI prevalence; furthermore, one patient reported unintentional weight loss more than 10 years after disease onset.

Unintentional weight loss was the most frequently reported symptom, also considering different mutations. The mechanisms behind weight loss remains largely unexplained, and a marked loss of weight has been observed in patients with hereditary ATTRv amyloidosis even before the onset of GI or other symptoms [7]. Unintentional weight loss could be considered as multifactorial; diarrhea and an increased metabolism, due to inflammatory reactions and oxidative stress, could be contributing factors $[7,17]$. 
Table II. Onset of GI symptoms in patients with hereditary transthyretin amyloidosis (ATTRv).

\begin{tabular}{|c|c|c|c|c|c|c|c|c|c|}
\hline & Val30Met & Phe64Leu & Glu89Gln & Ala120Ser & Thr59Lys & Ala109Ser & Val122Ile & Ile88Leu & Total \\
\hline Any gastro-intestinal symptoms & $15 / 16$ & $8 / 12$ & $3 / 4$ & $3 / 3$ & $1 / 1$ & $1 / 1$ & $0 / 1$ & $1 / 1$ & $32 / 39$ \\
\hline Onset $<5$ years & $15 / 15$ & $8 / 8$ & $3 / 3$ & $3 / 3$ & $1 / 1$ & $1 / 1$ & - & $1 / 1$ & $32 / 32$ \\
\hline Unintentional weight loss & $6 / 16$ & $6 / 12$ & $3 / 4$ & $3 / 3$ & $0 / 1$ & $0 / 1$ & $0 / 1$ & $0 / 1$ & $18 / 39$ \\
\hline Onset $<5$ years & $4 / 6$ & $5 / 6$ & $3 / 3$ & $3 / 3$ & - & - & - & - & $15 / 18$ \\
\hline Onset $5-10$ years & $1 / 6$ & $1 / 6$ & - & - & - & - & - & - & $2 / 18$ \\
\hline Onset $>10$ years & $1 / 6$ & - & - & - & - & - & - & - & $1 / 18$ \\
\hline Nausea & $4 / 16$ & $1 / 12$ & $0 / 4$ & $0 / 3$ & $0 / 1$ & $0 / 1$ & $0 / 1$ & $0 / 1$ & $5 / 39$ \\
\hline Onset $<5$ years & $2 / 4$ & $1 / 1$ & - & - & - & - & - & - & $3 / 5$ \\
\hline Onset $5-10$ years & $2 / 4$ & - & - & - & - & - & - & - & $2 / 5$ \\
\hline Early satiety & $3 / 16$ & $3 / 12$ & $0 / 4$ & $2 / 3$ & $1 / 1$ & $0 / 1$ & $0 / 1$ & $0 / 1$ & $9 / 39$ \\
\hline Onset $<5$ years & $2 / 3$ & $3 / 3$ & - & $2 / 2$ & - & - & - & - & $7 / 9$ \\
\hline Onset $5-10$ years & $1 / 3$ & - & - & - & $1 / 1$ & - & - & - & $2 / 9$ \\
\hline Vomiting & $2 / 16$ & $1 / 12$ & $0 / 4$ & $0 / 3$ & $1 / 1$ & $0 / 1$ & $0 / 1$ & $0 / 1$ & $4 / 39$ \\
\hline Onset $<5$ years & $1 / 2$ & $1 / 1$ & - & - & $1 / 1$ & - & - & - & $3 / 4$ \\
\hline Onset $5-10$ years & $1 / 2$ & - & - & - & - & - & - & - & $1 / 4$ \\
\hline Diarrhea & $3 / 16$ & $4 / 12$ & $3 / 4$ & $2 / 3$ & $1 / 1$ & $1 / 1$ & $0 / 1$ & $0 / 1$ & $14 / 39$ \\
\hline Onset $<5$ years & $2 / 3$ & $4 / 4$ & $3 / 3$ & $2 / 2$ & $1 / 1$ & $1 / 1$ & 0 & 0 & $13 / 14$ \\
\hline Onset $5-10$ years & $1 / 3$ & - & - & - & - & - & - & - & $1 / 14$ \\
\hline Constipation & $3 / 16$ & $1 / 12$ & $0 / 4$ & $2 / 3$ & $1 / 1$ & $0 / 1$ & $0 / 1$ & $1 / 1$ & $8 / 39$ \\
\hline Onset $<5$ years & $2 / 3$ & $1 / 1$ & - & $2 / 2$ & $1 / 1$ & - & - & $1 / 1$ & $7 / 8$ \\
\hline Onset $5-10$ years & $1 / 3$ & - & - & - & - & - & - & - & $1 / 8$ \\
\hline Diarrhea/constipation & $4 / 16$ & $2 / 12$ & $0 / 4$ & $3 / 3$ & $1 / 1$ & $0 / 1$ & $0 / 1$ & $0 / 1$ & $10 / 39$ \\
\hline Onset $<5$ years & $2 / 4$ & $2 / 2$ & - & $3 / 3$ & $1 / 1$ & - & - & - & $8 / 10$ \\
\hline Onset $5-10$ years & $2 / 4$ & - & - & - & - & - & - & - & $2 / 10$ \\
\hline Fecal incontinence & $0 / 16$ & $2 / 12$ & $0 / 4$ & $0 / 3$ & $0 / 1$ & $1 / 1$ & $0 / 1$ & $0 / 1$ & $3 / 39$ \\
\hline Onset $<5$ years & - & $2 / 2$ & - & - & - & $1 / 1$ & - & - & $3 / 3$ \\
\hline Abdominal pain & $2 / 16$ & $0 / 12$ & $0 / 4$ & $0 / 3$ & $0 / 1$ & $0 / 1$ & $0 / 1$ & $1 / 1$ & $3 / 39$ \\
\hline Onset $<5$ years & $2 / 2$ & - & - & - & - & - & - & $1 / 1$ & $3 / 3$ \\
\hline Abdominal bloating & $7 / 16$ & $1 / 12$ & $0 / 4$ & $1 / 3$ & $1 / 1$ & $0 / 1$ & $0 / 1$ & $1 / 1$ & $11 / 39$ \\
\hline Onset $<5$ years & $4 / 7$ & $1 / 1$ & - & $1 / 1$ & - & - & - & $1 / 1$ & $7 / 11$ \\
\hline Onset 5-10 years & $3 / 7$ & - & - & - & $1 / 1$ & - & - & - & $4 / 11$ \\
\hline
\end{tabular}

Diarrhea was the second most frequently reported manifestation in our analysis. The origin of this symptom is unclear and it is probably multifactorial. It is generally suggested that one of the contributing factors could be the presence of motility disturbances of the GI tract caused by an autonomic neuropathy [16]. Other factors could be malabsorption of fat and bile acids, as well as the frequent presence of bacterial overgrowth of the small bowel [16].

Abdominal bloating is also frequently reported, namely for patients with Val30Met mutation; many factors can contribute to this symptom, including possible bowel inflammation due to reaction against amyloid deposits [17].

\section{CONCLUSIONS}

Gastrointestinal symptoms were frequently reported by our patients, unintentional weight loss being the most prevalent. Patients with Val30Met mutation continued to develop further GI manifestations over time. Conversely, all non-Val30Met cases disclosed almost all GI symptoms within 5 years since disease onset. Better knowledge of GI manifestations of hereditary ATTRv amyloidosis and of their pathogenesis can provide novel targets for a more effective supportive treatment of these symptoms.

Conflicts of interest: M.L. received financial grants (honoraria and speaking) from Ackea, Alnylam and Pfizer, and travel grants from Ackea, Alnylam, Pfizer, Kedrion, Csl Behring, and Grifols. A.R. received travel grants from Pfizer and Csl Behring, and financial grant from Akcea. A.D.P. received travel grants from Pfizer. G.B. received financial grants (honoraria and speaking) from Alnylam, and travel grants from Pfizer, Alnylam and Grifols. A.G. received research grants and financial grants (honoraria and speaking) from: EISAI S.r.l., 3PSolutions, Real Time Meeting, Fondazione Istituto Danone,TAKEDA S.p.A., Advisory Board VSL3, Sinergie S.r.l. Board MRGE, Advisory Board EISAI Studio Reflect, ABBVIE "Paziente HCV", Sandoz S.p.A., SANOFI S.p.A.. P.C. received research grants from: Bayer, Schering, Biogen, BoehringerIngelheim, Eisai, Novartis, 
Lundbeck, Merck Sharp \& Dohme, Sanofi-Aventis, Sigma-Tau, and UCB Pharma. M.S. received financial grants (honoraria and speaking) from Ackea and Alnylam, and travel grants from Grifols. A.T. and V.G. have none potential conflicts of interest to be disclosed.

Authors' contribution: M.L. conceived and designed the study, analysis of data. A.T. revised the paper for important intellectual content, contributions to the study conception and design, analysis of data. A.R., A.D.P., V.G.: analysis of data. G.B., A.G., P.C.: revised the paper for important intellectual content. All authors critically revised the manuscript, approved the final version to be published, and agree to be accountable for all aspects of the work.

\section{REFERENCES}

1. Luigetti M, Romano A, Di Paolantonio A, Bisogni G, Sabatelli M Diagnosis and Treatment of Hereditary Transthyretin Amyloidosis (hATTR) Polyneuropathy: Current Perspectives on Improving Patient Care. Ther Clin Risk Manag 2020;16:109-123. doi:10.2147/TCRM. S219979

2. Benson MD, Kincaid JC. The molecular biology and clinical features of amyloid neuropathy. Muscle Nerve 2007;36:411-423. doi:10.1002/ mus. 20821

3. Adams D, Koike H, Slama M, Coelho T. Hereditary transthyretin amyloidosis: a model of medical progress for a fatal disease. Nat Rev Neurol 2019;15:387-404. doi:10.1038/s41582-019-0210-4

4. Conceição I, González-Duarte A, Obici L, et al. "Red-flag" symptom clusters in transthyretin familial amyloid polyneuropathy. J Peripher Nerv Syst 2016;21:5-9. doi:10.1111/jns.12153

5. Planté-Bordeneuve V, Said G. Familial amyloid polyneuropathy. Lancet Neurol 2011;10:1086-1097. doi:10.1016/S1474-4422(11)70246-0

6. Luigetti M, Conte A, Del Grande A, et al. TTR-related amyloid neuropathy: clinical, electrophysiological and pathological findings in 15 unrelated patients. Neurol Sci 2013;34:1057-1063. doi:10.1007/ s10072-012-1105-y
7. Wixner J, Mundayat R, Karayal ON, et al. THAOS: gastrointestina manifestations of transthyretin amyloidosis - common complications of a rare disease. Orphanet J Rare Dis 2014;9:61. doi:10.1186/17501172-9-61

8. Obici L, Suhr OB. Diagnosis and treatment of gastrointestinal dysfunction in hereditary TTR amyloidosis. Clin Auton Res 2019;29(Suppl 1):55-63. doi:10.1007/s10286-019-00628-6

9. Gertz MA. Hereditary ATTR amyloidosis: burden of illness and diagnostic challenges. Am J Manag Care 2017;23(7 Suppl):S107-S112.

10. Coelho T, Maia LF, Martins da Silva A, et al. Tafamidis for transthyretin familial amyloid polyneuropathy: a randomized, controlled trial. Neurology 2012;79:785-792. doi:10.1212/WNL.0b013e3182661eb1

11. Adams D, Gonzalez-Duarte A, O'Riordan WD, et al. Patisiran, an RNAi Therapeutic, for Hereditary Transthyretin Amyloidosis. N Engl J Med 2018;379:11-21. doi:10.1056/NEJMoa1716153

12. Benson MD, Waddington-Cruz M, Berk JL, et al. Inotersen Treatment for Patients with Hereditary Transthyretin Amyloidosis. N Engl J Med 2018;379:22-31. doi:10.1056/NEJMoa1716793

13. Maurer MS, Schwartz JH, Gundapaneni B, et al. Tafamidis Treatment for Patients with Transthyretin Amyloid Cardiomyopathy. N Engl J Med 2018;379:1007-1016. doi:10.1056/NEJMoa1805689

14. González-Duarte A, Berk JL, Quan D, et al. Analysis of autonomic outcomes in APOLLO, a phase III trial of the RNAi therapeutic patisiran in patients with hereditary transthyretin-mediated amyloidosis. J Neurol 2020;267:703-712. doi:10.1007/s00415-019-09602-8

15. Luigetti M, Bisogni G, Romano A, et al. Sudoscan in the evaluation and follow-up of patients and carriers with TTR mutations: experience from an Italian Centre. Amyloid 2018;25:242-246. doi:10.1080/13506 129.2018.1545640

16. Nakov R, Sarafov S, Nakov V, et al. Gastrointestinal Manifestations in Hereditary Transthyretin Amyloidosis associated with Glu89Gln Mutation. J Gastrointestin Liver Dis 2019;28:421-426. doi:10.15403/jgld-362

17. Nakov R, Sarafov S, Nakov V, et al. Fecal calprotectin levels are elevated in transthyretin amyloidosis patients with gastrointestinal manifestations. Medicine (Baltimore) 2020;99:e19509. doi:10.1097/ MD.0000000000019509 